\title{
Acupuncture for post-stroke depression: a systematic review and meta-analysis
}

\author{
Ran Liu ${ }^{1,2}$, Kun Zhang ${ }^{1,2}$, Qiu-yu Tong ${ }^{1}$, Guang-wei Cui ${ }^{1,2}$, Wen Ma ${ }^{1 *}$ and Wei-dong Shen ${ }^{1,2^{*}}$ (D)
}

\begin{abstract}
Background: Acupuncture for post-stroke depression (PSD) has been evolving, but uncertainty remains. To assess the existing evidence from randomized clinical trials (RCTs) of acupuncture for PSD, we sought to draw conclusions by synthesizing RCTs.

Methods: An exhaustive literature search was conducted in seven electronic databases from their inception dates to April 19, 2020, to identify systematic reviews (SRs) and meta-analyses (MAs) on this topic. The primary RCTs included in the SRs/MAs were identified. We also conducted a supplementary search for RCTs published from January 1, 2015, to May 12, 2020. Two reviewers extracted data separately and pooled data using RevMan 5.3 software. The quality of evidence was critically appraised with the Grades of Recommendation, Assessment, Development and Evaluation (GRADE) system.
\end{abstract}

Results: A total of 17 RCTs involving 1402 patients were included. Meta-analysis showed that participants who received a combination of acupuncture and conventional treatments exhibited significantly lower scores on the HAM-D ${ }_{17}$, HAM-D 24 and HAM-D (MD, -5.08 [95\% Cl, -6.48 to -3.67$\left.], I^{2}=0 \%\right),(\mathrm{MD},-9.72[95 \% \mathrm{Cl},-14.54$ to 4.91], $\left.P^{2}=65 \%\right)$ and $(\mathrm{MD},-2.72[95 \% \mathrm{Cl},-3.61$ to -1.82$]$, respectively) than those who received conventional treatment. However, there was no significant difference in acupuncture versus antidepressants in terms of the 17item, 24-item and HAM-D scales (MD, -0.43 [95\% Cl, - 1.61 to 0.75$\left.], P^{2}=51 \%\right),(\mathrm{MD},-3.09$ [95\% Cl, -10.81 to 4.63], $\left.P^{2}=90 \%\right)$ and $\left(\mathrm{MD},-1.55[95 \% \mathrm{Cl},-4.36\right.$ to 1.26$], P^{2}=95 \%$, respectively). For adverse events, acupuncture was associated with fewer adverse events than antidepressants (RR, $0.16[95 \% \mathrm{Cl}, 0.07$ to 0.39$], P=35 \%$ ), but there was no significant difference in the occurrence of adverse events between the combination of acupuncture and conventional treatments versus conventional treatments ( $\mathrm{RR}, 0.63[95 \% \mathrm{Cl}, 0.21$ to 1.83$], l^{2}=38 \%$ ). The quality of evidence was low to very low due to the substantial heterogeneity among the included studies.

Conclusions: The current review indicates that acupuncture has greater effect on PSD and better safety profile than antidepressants, but high-quality evidence evaluating acupuncture for PSD is still needed.

Keywords: Acupuncture, Post-stroke depression, Systematic review, Meta-analysis, Effectiveness, Safety

\section{Background}

Stroke is the second largest cause of mortality and disability in the world according to the Global Burden of Diseases, Injuries, and Risk Factors Study (GBD), contributing to a high burden of disease [1]. Approximately

\footnotetext{
* Correspondence: 13524650702@126.com; shenweidong@shutcm.edu.cn 'Department of Acupuncture, Shuguang Hospital, Shanghai University of Traditional Chinese Medicine, Shanghai 201203, China

Full list of author information is available at the end of the article
}

$31.1 \%$ of stroke survivors will develop post-stroke depression (PSD), which is characterized by long-term persistent depression, often complicating the course of stroke and adversely affecting functional recovery and quality of life [2-6]. In addition, PSD could also raise the risk of mortality in stroke survivors and threaten the life security of these patients [7]. Currently, antidepressants, primarily 5-hydroxytryptamine $(5-\mathrm{HT})$ reuptake inhibitors (SSRIs), are used clinically to prevent and treat

C C The Author(s). 2021 Open Access This article is licensed under a Creative Commons Attribution 4.0 International License, which permits use, sharing, adaptation, distribution and reproduction in any medium or format, as long as you give appropriate credit to the original author(s) and the source, provide a link to the Creative Commons licence, and indicate if changes were made. The images or other third party material in this article are included in the article's Creative Commons licence, unless indicated otherwise in a credit line to the material. If material is not included in the article's Creative Commons licence and your intended use is not permitted by statutory regulation or exceeds the permitted use, you will need to obtain permission directly from the copyright holder. To view a copy of this licence, visit http://creativecommons.org/licenses/by/4.0/ The Creative Commons Public Domain Dedication waiver (http://creativecommons.org/publicdomain/zero/1.0/) applies to the data made available in this article, unless otherwise stated in a credit line to the data. 
PSD, but the risk of cerebral haemorrhage and recurrent stroke associated with this approach cannot be ignored $[8,9]$. Therefore, there is an urgent need for innovative strategies including more effective and secure intervention in PSD [10].

Acupuncture is widely used in the treatment of PSD, and the number of studies on acupuncture for PSD has been recently increasing. Although more than 5 systematic reviews (SRs) and meta-analyses (MAs) [11-15] were conducted to appraisal the effect of acupuncture on PSD, none arrived at a consistent conclusion. The reason for that is the wide variations in interventions, qualities, and outcomes selected in these studies. For example, some of these SRs and MAs included randomized controlled trials (RCTs) with acupuncture, acupressure, acupoint injection or moxibustion, which could increase the risk of heterogeneity. In addition, some SRs/MAs failed to report outcomes according to different criteria, such as different versions of the Hamilton Rating Scale for Depression (HAM-D) or different definitions of effective rate and curative rate, which might cause estimate instability during the evaluation process. In addition, more recent trials about acupuncture for PSD have been published and add to the evidence of the association between acupuncture and the degree of depression. A trial conducted in 2018 revealed that patients with moderate or severe depression who received a combination of acupuncture and medication experienced visible depression relief [16]. This reduction in depression was also certified in a 2019 study [17].

In light of the growing number of studies of acupuncture for PSD and the ensuing need for critical evaluation, we conducted a new MA and used the GRADE system to assess the evidence quality for the outcomes of interest to discover the true efficacy and gaps in this field and to provide recommendations for clinical practice.

\section{Methods}

This MA was performed according to the Cochrane Handbook for Systematic Reviews of Interventions [18] and presented based on Preferred Reporting Items for Systematic Reviews and Meta-analyses guidelines [19]. This protocol was registered with the International Platform of Registered Systematic Review and Meta-Analysis Protocols (INPLASY202050117).

\section{Literature search}

Two researchers (W Ma and R Liu) searched the PubMed, Cochrane Library, EMBASE, China National Knowledge Infrastructure (CNKI), WANFANG DATA, Chinese Biomedical Literature (CBM), and Chongqing VIP (CQVIP) databases from their respective inception dates to April 19, 2020, using the keywords acupuncture, electroacupuncture and post-stroke depression to recognize published SRs and MAs evaluating the association between acupuncture and PSD. There were no language or study-blinding restrictions (detailed search strategies for PubMed are reported in Supplemental Table 1). We identified original randomized clinical trials (RCTs) included in the SRs/MAs. An additional search was conducted to identify recently published RCTs, from January 1, 2015, to May 12, 2020, meeting the inclusion criteria using the same databases and search strategy as described above (detailed search strategies for PubMed are reported in Supplemental Table 2).

\section{Inclusion and exclusion criteria}

We abided by the Participants, Interventions, Comparisons, Outcomes and Study Design (PICOS) approach to establish the inclusion criteria, which were as follows: (1) Patients included were diagnosed with PSD with no restrictions on diagnostic criteria, gender, age, race, onset time or source of cases and regardless of the cause of the stroke. (2) Eligible interventions in the treatment group included acupuncture, electroacupuncture regardless of needling techniques, stimulation methods and acupoint selection. The control group was treated with sham acupuncture or conventional treatments (e.g., antidepressants, basic treatments, psychological counselling and physical rehabilitation). (3) The comparison reported was acupuncture/electroacupuncture vs. placebo/ sham acupuncture, acupuncture/electroacupuncture vs. antidepressants, or acupuncture/electroacupuncture + conventional treatments vs. conventional treatments. (4) Degree of depression was selected as the targeted outcome because of its crucial role in PSD assessment [20]. The primary outcome included the HAM-D score, while the secondary outcome was adverse events. (5) The study was a RCT evaluating acupuncture for PSD.

The exclusion criteria were as follows: (1) duplicates, (2) literature with incorrect data or inaccessible data, and (3) Chinese trials that were not published in the Chinese core journal of Peking University (PKU).

\section{Study selection}

There were two steps in the study selection process. First, we identified the eligible RCTs included in the SRs/MAs. Second, we recognized the RCTs of interest from the supplemental search. Both processes were performed by creating a database using Noteexpress 3.2.0 software, and after eliminating duplicates, we read the titles and abstracts for a preliminary screening. When studies could not be definitively excluded based on the titles and abstracts, we downloaded and the full text documents until all RCTs were confirmed. Two researchers (K Zhang and GW Cui) selected the literature, 
and disagreements were resolved by another reviewer $(\mathrm{R}$ Liu) when they had different opinions.

\section{Data extraction}

Two researchers (GW Cui and QY Tong) independently extracted the following information using predesigned forms: lead author, publication year, details of intervention measurement, sample size and dropout, ages of the participants, degree of depression, depression duration, outcomes and adverse events. If the trials had more than two groups or permitted multiple comparisons, we extracted only the information and data of interest reported in the original articles. Any divergence was resolved by consensus (W Ma).

\section{Risk of bias assessment}

The methodological quality of the included studies was assessed by two researchers (R Liu and K Zhang) according to the Cochrane handbook 5.1.0 [18], and the studies were ranked as high, unclear, and low risk in seven domains, namely randomization sequence generation, allocation concealment, blinding of participants and personnel, blinding of outcome assessment, incomplete outcome data, selective reporting and other bias. If necessary, we contacted the study authors when relevant information could not be identified from the articles or supplementary materials. In addition, we graded the overall quality of the included trials as high, moderate, or low quality according to previously published highquality literature [21]. Disagreements were resolved through discussion with a third researcher (WD Shen).

\section{GRADE evaluation}

The GRADE system [22] was used to evaluate the evidence quality of the evaluated outcomes. For each outcome, we automatically awarded each study four points, as these were RCTs, and they were downgraded if there were increased risks of bias, inconsistency, indirectness, inaccuracy, and publication bias. We classified evidence quality as A (high), B (moderate), C (low), or D (very low). Two researchers (R Liu and QY Tong) independently assessed the quality of evidence and resolved any disputes through discussions with a third researcher (WD Shen).

\section{Statistical analysis}

We performed a meta-analysis of the RCTs with available data to calculate the effect size and 95\% confidence interval $(\mathrm{CI})$ using the random effects model based on significant heterogeneity $\left(P<0.1\right.$ and $\left.I^{2}>50 \%\right)$. The HAM-D scale is presented as the mean difference (MD), as it is a continuous variable, and adverse events are presented as relative risks (RRs), as they are dichotomous variables. Statistical analyses were performed with
RevMan 5.3 software. Two-sided $P<0.05$ was considered statistically significant.

When possible and appropriate, planned subgroup analyses using the same models were conducted to identify the source of heterogeneity and reliability of the various results. Sensitivity analysis was performed by excluding low-quality studies and trials evaluating sham acupuncture. Publication bias was assessed by examining funnel plots for asymmetry when at least 10 articles were included.

\section{Results}

\section{Literature selection}

From the searches for SRs and MAs, 1037 potentially eligible records were identified. Titles and abstracts of these records were screened for exclusion. Full texts of 38 records were read, and 11 SRs/MAs that contained 167 RCTs met the inclusion criteria. After 141 RCTs were excluded for being a duplicate or being unpublished in the PKU journal, we obtained the full texts of 26 potential candidate RCTs. Among these, we ultimately included 13 articles. Supplemental Table 3 summarizes the RCTs included in the SRs/MAs.

A total of 2615 articles were identified in a recent 5year supplemental search; of these, 28 eligible full texts were reviewed after excluding duplicates and irrelevant articles. Seven trials met the inclusion criteria in this process. Finally, after excluding 3 duplicate trials, 17 RCTs involving 1402 participants were included in this review (Fig. 1). We excluded 33 trials for the reasons listed in Supplemental Table 4.

\section{Characteristics of the included studies}

Quantitative synthesis was performed with 17 RCTs [16, $17,23-37$ ] by pooling the results in this review, and all trials were implemented in China. Sample sizes ranged from 10 to 145 participants, and a total of 1402 patients were included, with 683 (48.7\%) in the experimental group and $719(51.3 \%)$ in the control group. Seven trials $[16,17,23,26,28,35,37]$ compared acupuncture or electroacupuncture combined with conventional treatment with conventional treatment, 11 studies [24, 25, 27, 29-34, 36, 37] compared acupuncture or electroacupuncture with antidepressants, and $2[30,33]$ adopted a 2 -group parallel contrast design and compared the combination of real acupuncture and oral placebo with the combination of antidepressants and sham acupuncture. One study [37] was a 3-group design containing acupuncture alone, acupuncture combined with antidepressants and antidepressants alone. With regard to the degree of depression [38], 7 of the RCTs [23, 24, 26, 28, $31,32,35]$ recruited PSD participants with potential mild, moderate and severe depression, 10 studies [16, 17, $25,27,29,30,33,34,36,37]$ enrolled patients with 


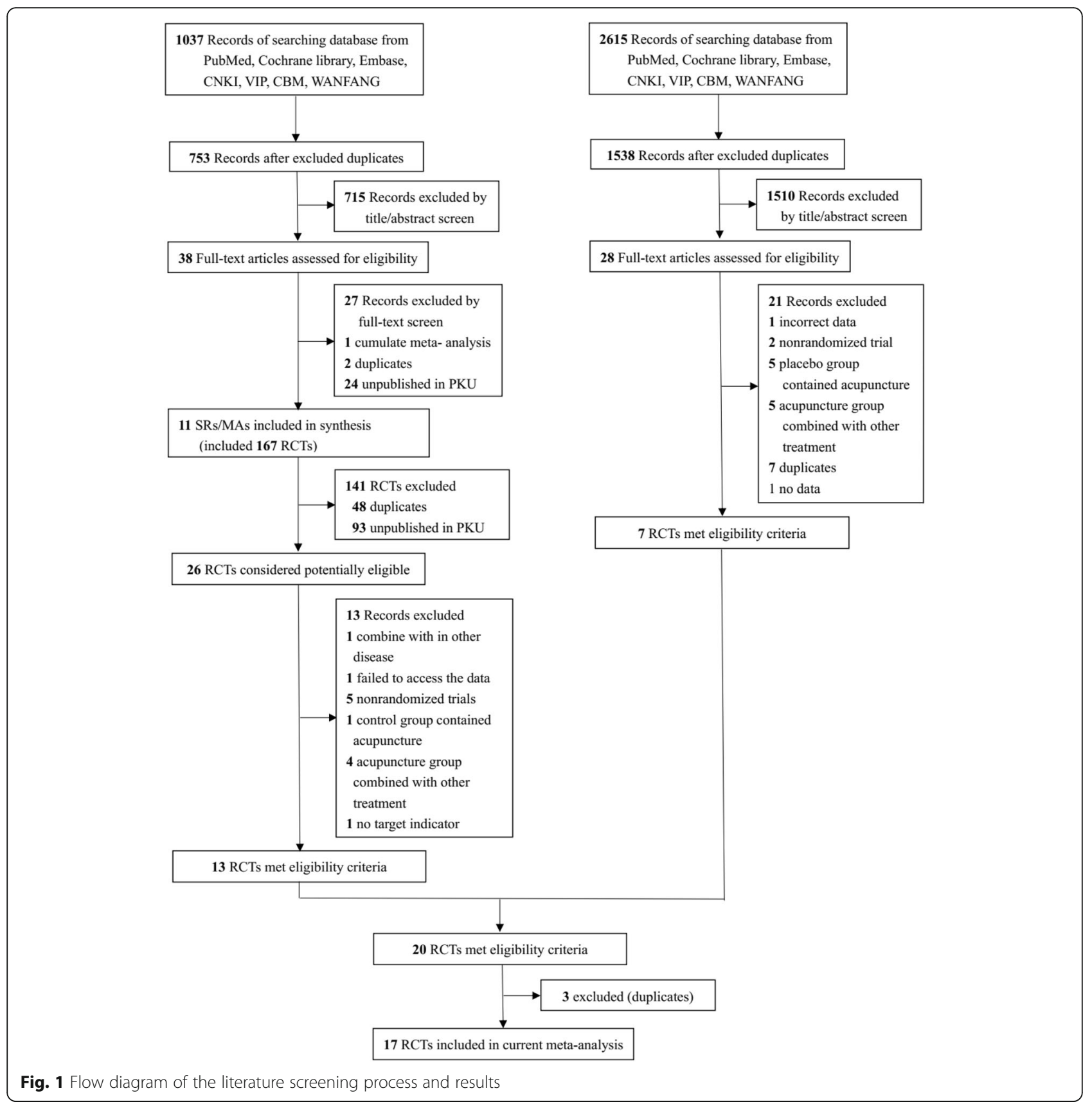

moderate and severe depression. The treatment durations ranged from 4 weeks to 6 months. The details of the duration, frequency, and number of sessions of the acupuncture programs are presented in the supplementary materials (Tables S6 $\sim$ S8). For outcomes, we only evaluated the HAM-D scale because it is considered the "Gold Standard" in depression assessment [20]. Seven studies $[25,26,28,30,33,34,37]$ used the HAM-D scale-17 items $\left(\mathrm{HAM}-\mathrm{D}_{17}\right)$ to evaluate the degree of depression, while four studies [16, 24, 27, 35] used the HAM-D scale-24 items (HAM- $\left.\mathrm{D}_{24}\right)$ as their outcome indicator. In addition, six trials $[17,23,29,31,32,36]$ did not report which version of the HAM-D they used. Adverse events are also an indispensable indicator to assess the safety of acupuncture. In this review, 10 studies [16, 17, 23, 29-31, 33, 34, $36,37]$ reported adverse events; among them, three trials $[16,23,36]$ found no obvious adverse events during the intervention, and one [33] was excluded because it presented overlapping data. Table 1 shows the details and characteristics of the included RCTs.

\section{Risk of bias assessment}

Fifteen studies [16, 17, 23, 25-35, 37] were rated as low risk because they reported specific randomization 
Table 1 Characteristics of RCTs Included in the Review and Summary of Trial Quality Assessment

\begin{tabular}{|c|c|c|c|c|c|c|c|c|c|c|}
\hline \multirow[t]{2}{*}{ Source } & \multirow{2}{*}{$\begin{array}{l}\text { Reference } \\
\text { No. }\end{array}$} & \multicolumn{2}{|l|}{ Intervention } & \multirow{2}{*}{$\begin{array}{l}\text { Sample } \\
\text { Size (T/ } \\
\text { C), } \\
\text { Dropout } \\
\text { (T/C) }\end{array}$} & \multirow[t]{2}{*}{ Age (T/C) } & \multirow{2}{*}{$\begin{array}{l}\text { Depression } \\
\text { Definition }\end{array}$} & \multirow[t]{2}{*}{ Duration } & \multirow{2}{*}{$\begin{array}{l}\text { Outcome } \\
\text { (s) }\end{array}$} & \multirow[t]{2}{*}{ Adverse events } & \multirow{2}{*}{$\begin{array}{l}\text { Overall } \\
\text { quality } \\
\text { for each } \\
\text { trial }\end{array}$} \\
\hline & & Treatment & Control & & & & & & & \\
\hline \multicolumn{11}{|c|}{ Acupuncture/EA + conventional treatment ${ }^{\mathrm{a}}$ vs. conventional treatment } \\
\hline $\begin{array}{l}\text { Guo } \\
2009\end{array}$ & [26] & $\begin{array}{l}\text { Acupuncture } \\
\text { (SP4, PC6, SJ5, } \\
\text { GB41, SI3, } \\
\text { BL62, LU7, KI6, } \\
30 \text { min/d) + } \\
\text { psychological } \\
\text { counselling+ } \\
\text { physical } \\
\text { rehabilitation }\end{array}$ & $\begin{array}{l}\text { Sertraline (50 } \\
\mathrm{mg} / \mathrm{d})+ \\
\text { psychological } \\
\text { counselling + } \\
\text { physical } \\
\text { rehabilitation }\end{array}$ & $40 / 40$ & $\begin{array}{l}65.8 \pm 9.61 / \\
67.6 \pm 12.43\end{array}$ & $\begin{array}{l}\operatorname{HAM}-\mathrm{D}_{17} \geq \\
8\end{array}$ & $30 d$ & HAM-D 17 & $N R$ & $\begin{array}{l}\text { Moderate } \\
\text { quality }\end{array}$ \\
\hline $\begin{array}{l}\text { Guo } \\
2012\end{array}$ & [28] & $\begin{array}{l}\text { Acupuncture } \\
\text { (PC6, GV 20, } \\
\text { GV29, LR3, } \\
\text { HT7, BL15, } \\
\text { SP6, KI3, ST36, } \\
\text { EX-HN1, ST40, } \\
30 \text { min/d) + } \\
\text { physical } \\
\text { rehabilitation }\end{array}$ & $\begin{array}{l}\text { physical } \\
\text { rehabilitation }\end{array}$ & $55 / 55$ & $\begin{array}{l}60.30 \pm \\
4.80 / \\
60.80 \pm 3.90\end{array}$ & $\begin{array}{l}\mathrm{HAM}_{8}-\mathrm{D}_{17} \geq \\
\end{array}$ & $30 d$ & HAM-D 17 & $N R$ & $\begin{array}{l}\text { Moderate } \\
\text { quality }\end{array}$ \\
\hline $\begin{array}{l}\text { Zhang } \\
2019\end{array}$ & [17] & $\begin{array}{l}\text { Acupuncture } \\
\text { (LI4, ST40, } \\
\text { ST16, HT7, } \\
\text { SP6, LR3, PC6, } \\
\text { EX-HN1, GV29, } \\
\text { GV24, GV20, } \\
40 \mathrm{~min} / \mathrm{d})+ \\
\text { Fluoxetine } \\
\text { (20 mg/d) }\end{array}$ & $\begin{array}{l}\text { Fluoxetine ( } 20 \\
\mathrm{mg} / \mathrm{d})\end{array}$ & $48 / 48$ & $\begin{array}{l}57.2 \pm 8.5 / \\
58.5 \pm 7.8\end{array}$ & HAM-D $\geq 20$ & $6 m$ & $\begin{array}{l}\text { HAM-D } \\
\text { scores }\end{array}$ & $\begin{array}{l}\text { T: } 2 \text { cases of skin } \\
\text { redness and } \\
\text { swelling, } 1 \text { case } \\
\text { of constipation. } \\
\text { C: } 1 \text { case of } \\
\text { constipation, } 1 \\
\text { case of } \\
\text { abdominal } \\
\text { distension. }\end{array}$ & $\begin{array}{l}\text { Moderate } \\
\text { quality }\end{array}$ \\
\hline $\begin{array}{l}\text { Sun } \\
2015\end{array}$ & [35] & $\begin{array}{l}\text { Acupuncture } \\
\text { (GV 20, GV16, } \\
\text { GV 24, GV 26, } \\
\text { GV14, GV 11, } \\
40 \text { min/d) + } \\
\text { Fluoxetine } \\
\text { (20 mg/d) }\end{array}$ & $\begin{array}{l}\text { Fluoxetine ( } 20 \\
\mathrm{mg} / \mathrm{d})\end{array}$ & $33 / 30$ & $\begin{array}{l}59 \pm 7 / 51 \pm \\
6\end{array}$ & $\begin{array}{l}\mathrm{HAM}-\mathrm{D}_{24} \geq \\
8\end{array}$ & $4 w$ & $\mathrm{HAM}-\mathrm{D}_{24}$ & $N R$ & $\begin{array}{l}\text { Moderate } \\
\text { quality }\end{array}$ \\
\hline $\begin{array}{l}\text { Chen } \\
2018\end{array}$ & [16] & $\begin{array}{l}\text { Acupuncture } \\
\text { (PC6, GV20, } \\
\text { LI4, KI3, LR3, } \\
\text { HT7, BL15, } \\
\text { SP6, KI3, ST36, } \\
\text { EX-HN1, ST10, } \\
30 \text { min/d) + } \\
\text { conventional } \\
\text { drug therapy }\end{array}$ & $\begin{array}{l}\text { Conventional } \\
\text { drug therapy }\end{array}$ & $30 / 30$ & $\begin{array}{l}57 \pm 11 / \\
58 \pm 11\end{array}$ & $\begin{array}{l}\mathrm{HAM}-\mathrm{D}_{24} \geq \\
24\end{array}$ & $4 w$ & $\mathrm{HAM}-\mathrm{D}_{24}$ & None & $\begin{array}{l}\text { Moderate } \\
\text { quality }\end{array}$ \\
\hline $\begin{array}{l}\text { Jiang } \\
2007\end{array}$ & [23] & $\begin{array}{l}\text { EA (GV20, EX- } \\
\text { HN3, sparse- } \\
\text { dense waves } \\
2 / 100 ~ H z \text {, tol- } \\
\text { erable } \\
\text { strength, } 30 \\
\text { min/d) + Flu- } \\
\text { oxetine (20 } \\
\text { mg/d) }\end{array}$ & $\begin{array}{l}\text { Fluoxetine ( } 20 \\
\mathrm{mg} / \mathrm{d})\end{array}$ & $\begin{array}{l}31 / 30,3 / \\
2\end{array}$ & $\begin{array}{l}60.32 \pm \\
3.26 / 61 \\
18 \pm 2.94\end{array}$ & $\begin{array}{l}\text { HAM-D } \\
\text { scores } \geq 8\end{array}$ & $28 d$ & $\begin{array}{l}\text { HAM-D } \\
\text { scores }\end{array}$ & None & $\begin{array}{l}\text { Moderate } \\
\text { quality }\end{array}$ \\
\hline $\begin{array}{l}\text { Sun Y } \\
\text { b } 2015\end{array}$ & [37] & $\begin{array}{l}\text { EA (the } \\
\text { midnight- } \\
\text { noon ebb- } \\
\text { flow theory, } \\
\text { continuous } \\
\text { wave, } 2 \mathrm{~Hz} \text {, } \\
30 \mathrm{~min} / \mathrm{d} \text { ) + }\end{array}$ & $\begin{array}{l}\text { Fluoxetine } \\
(20 \mathrm{mg} / \mathrm{d})\end{array}$ & $31 / 31$ & $\begin{array}{l}67 \pm 4 / 69 \pm \\
5\end{array}$ & $\begin{array}{l}\text { HAM-D }{ }_{17} \geq \\
17\end{array}$ & $6 \mathrm{~m}$ & HAM-D 17 & $\begin{array}{l}\text { T: } 1 \text { case of dry } \\
\text { mouth, } 1 \text { case of } \\
\text { fatigue. } \\
\text { C: } 2 \text { cases of } \\
\text { intermittent } \\
\text { headache, } 2 \\
\text { cases of loss of }\end{array}$ & $\begin{array}{l}\text { High } \\
\text { quality }\end{array}$ \\
\hline
\end{tabular}


Table 1 Characteristics of RCTs Included in the Review and Summary of Trial Quality Assessment (Continued)

\begin{tabular}{|c|c|c|c|c|c|c|c|c|c|c|}
\hline \multirow[t]{2}{*}{ Source } & \multirow{2}{*}{$\begin{array}{l}\text { Reference } \\
\text { No. }\end{array}$} & \multicolumn{2}{|l|}{ Intervention } & \multirow{2}{*}{$\begin{array}{l}\text { Sample } \\
\text { Size (T/ } \\
\text { C), } \\
\text { Dropout } \\
\text { (T/C) } \\
\end{array}$} & \multirow[t]{2}{*}{ Age $(T / C)$} & \multirow{2}{*}{$\begin{array}{l}\text { Depression } \\
\text { Definition }\end{array}$} & \multirow[t]{2}{*}{ Duration } & \multirow{2}{*}{$\begin{array}{l}\text { Outcome } \\
\text { (s) }\end{array}$} & \multirow[t]{2}{*}{ Adverse events } & \multirow{2}{*}{$\begin{array}{l}\text { Overall } \\
\text { quality } \\
\text { for each } \\
\text { trial }\end{array}$} \\
\hline & & Treatment & Control & & & & & & & \\
\hline & & $\begin{array}{l}\text { Fluoxetine } \\
(20 \mathrm{mg} / \mathrm{d})\end{array}$ & & & & & & & $\begin{array}{l}\text { appetite, } 2 \text { cases } \\
\text { of nausea. }\end{array}$ & \\
\hline \multicolumn{11}{|c|}{ Acupuncture/EA (+placebo) vs. antidepressants (+sham acupuncture) } \\
\hline $\begin{array}{l}\text { Zhou } \\
2012\end{array}$ & {$[24]$} & $\begin{array}{l}\text { Acupuncture } \\
\text { (GV20, Ex- } \\
\text { HN1, LR3, } \\
\text { ST36, SP6, } 20 \\
\text { min/d) }\end{array}$ & $\begin{array}{l}\text { Fluoxetine } \\
(20 \mathrm{mg} / \mathrm{d})\end{array}$ & $\begin{array}{l}30 / 30,2 / \\
2\end{array}$ & $\begin{array}{l}65.34 \pm \\
10.60 / \\
66.03 \pm 9.51\end{array}$ & $\begin{array}{l}\mathrm{HAM}-\mathrm{D}_{24} \geq \\
8\end{array}$ & $30 \mathrm{~d}$ & $\mathrm{HAM}^{-\mathrm{D}_{24}}$ & NR & $\begin{array}{l}\text { Low } \\
\text { quality }\end{array}$ \\
\hline $\begin{array}{l}\text { Sun } \\
2013\end{array}$ & [27] & $\begin{array}{l}\text { Acupuncture } \\
\text { (GV20, GV16, } \\
\text { GV24, GV26, } \\
\text { GV14, GV11, } \\
40 \text { min/d) }\end{array}$ & $\begin{array}{l}\text { Fluoxetine } \\
(20 \mathrm{mg} / \mathrm{d})\end{array}$ & $30 / 30$ & $\begin{array}{l}58 \pm 8 / 59 \pm \\
9\end{array}$ & $\begin{array}{l}\operatorname{HAM}-\mathrm{D}_{24} \geq \\
20\end{array}$ & $4 w$ & $\mathrm{HAM}-\mathrm{D}_{24}$ & NR & $\begin{array}{l}\text { Moderate } \\
\text { quality }\end{array}$ \\
\hline $\begin{array}{l}\text { Ding } \\
2003\end{array}$ & {$[29]$} & $\begin{array}{l}\text { Acupuncture } \\
\text { (GV20, DU24, } \\
\text { DU16, } 30 \mathrm{~min} / \\
\text { d) }\end{array}$ & $\begin{array}{l}\text { Fluoxetine } \\
\text { (20 mg/d) }\end{array}$ & $\begin{array}{l}30 / 30,1 / \\
1\end{array}$ & NR & $\begin{array}{l}\text { HAM-D } \\
\text { score } \geq 20\end{array}$ & $60 d$ & $\begin{array}{l}\text { HAM-D } \\
\text { score }\end{array}$ & $\begin{array}{l}\text { C: } 3 \text { cases of } \\
\text { mild abdominal } \\
\text { pain, } 2 \text { cases of } \\
\text { mild nausea and } \\
\text { vomiting, } 3 \\
\text { cases of elevated } \\
\text { GOT and GPT. }\end{array}$ & $\begin{array}{l}\text { Moderate } \\
\text { quality }\end{array}$ \\
\hline Li 2011 & {$[30]$} & $\begin{array}{l}\text { Acupuncture } \\
\text { (GV20, Ex- } \\
\text { HN1, LR3, Ex- } \\
\text { HN3, } 30 \mathrm{~min} / \\
\text { d) + oral } \\
\text { placebo }\end{array}$ & $\begin{array}{l}\text { Non-acupoint } \\
\text { spots+ } \\
\text { Fluoxetine } \\
(20 \mathrm{mg} / \mathrm{d})\end{array}$ & $\begin{array}{l}23 / 20,1 / \\
2\end{array}$ & $\begin{array}{l}29-90 / 32- \\
63\end{array}$ & $\begin{array}{l}\operatorname{HAM}_{17}-D_{17} \geq \\
\end{array}$ & $6 w$ & HAM-D 17 & $\begin{array}{l}\text { T: } 1 \text { case of pain, } \\
1 \text { case of } \\
\text { dizziness and } \\
\text { nausea and } 1 \\
\text { case of } \\
\text { haematoma. C: } 1 \\
\text { case of dizziness, } \\
1 \text { case of } \\
\text { numbness, } 1 \\
\text { case of } \\
\text { palpitation. }\end{array}$ & $\begin{array}{l}\text { High } \\
\text { quality }\end{array}$ \\
\hline $\begin{array}{l}\text { Liu } \\
2006\end{array}$ & {$[32]$} & $\begin{array}{l}\text { Acupuncture } \\
\text { (Ex-HN1, PC6, } \\
\text { HT7, ST36, } \\
\text { SP6, KI6, LR3, } \\
\text { BL62, } 30 \text { min/ } \\
\text { d) }\end{array}$ & $\begin{array}{l}\text { Fluoxetine } \\
(20 \mathrm{mg} / \mathrm{d})\end{array}$ & $101 / 145$ & $\begin{array}{l}60.0 \pm 9.8 / \\
59.6 \pm 8.9\end{array}$ & $\begin{array}{l}\text { HAM-D } \\
\text { scores } \geq 8\end{array}$ & $6 w$ & $\begin{array}{l}\text { HAM-D } \\
\text { score }\end{array}$ & NR & $\begin{array}{l}\text { Moderate } \\
\text { quality }\end{array}$ \\
\hline $\begin{array}{l}\text { Qian } \\
2015\end{array}$ & {$[33]$} & $\begin{array}{l}\text { Acupuncture } \\
\text { (GV26, PC6, } \\
\text { HT7, ST36, } 30 \\
\text { min/d) + } \\
\text { placebo }\end{array}$ & $\begin{array}{l}\text { Fluoxetine } \\
(20 \mathrm{mg} / \mathrm{d})+ \\
\text { Shallow } \\
\text { puncture }\end{array}$ & $\begin{array}{l}32 / 33,2 / \\
1\end{array}$ & $\begin{array}{l}67.59 \pm \\
11.02 / \\
67.74 \pm 8.61\end{array}$ & $\begin{array}{l}\operatorname{HAM}_{17} \mathrm{D}_{17} \geq \\
\end{array}$ & $6 w$ & HAM-D 17 & $\begin{array}{l}\text { T: } 2 \text { cases of } \\
\text { needle sticks, } 1 \\
\text { case of bent } \\
\text { needles, } 2 \text { cases } \\
\text { of haematomas. } \\
\text { C: } 1 \text { case of } \\
\text { haematomas. }\end{array}$ & $\begin{array}{l}\text { High } \\
\text { quality }\end{array}$ \\
\hline $\begin{array}{l}\text { Zhang } \\
2016\end{array}$ & {$[34]$} & $\begin{array}{l}\text { Acupuncture } \\
\text { (GV26, GV24, } \\
\text { GV15, BL18, } \\
\text { BL23, LR3, KI3, } \\
\text { PC6, HT7, } \\
\text { CV17, } 15 \mathrm{~min} / \\
\text { d) }\end{array}$ & $\begin{array}{l}\text { Escitalopram } \\
\text { Oxalate tablet } \\
(1.25-10 \mathrm{mg} / \\
\text { d) }\end{array}$ & $\begin{array}{l}33 / 32,2 / \\
3\end{array}$ & $\begin{array}{l}58.0 \pm 5.3 / \\
59.0 \pm 4.5\end{array}$ & $\begin{array}{l}\operatorname{HAM}_{18} \mathrm{D}_{17} \geq \\
\end{array}$ & $8 w$ & HAM-D 17 & $\begin{array}{l}\text { C: } 2 \text { cases of } \\
\text { dizziness, } 2 \text { cases } \\
\text { of nausea and } 1 \\
\text { case of tolerable } \\
\text { joint pain }\end{array}$ & $\begin{array}{l}\text { Moderate } \\
\text { quality }\end{array}$ \\
\hline $\begin{array}{l}\text { Zhou } \\
2016\end{array}$ & [36] & $\begin{array}{l}\text { Acupuncture } \\
\text { (GV20, EX- } \\
\text { HN1, HT7, } \\
\text { SP6, PC6, } \\
\text { GV24, } 30 \mathrm{~min} / \\
\text { d) }\end{array}$ & $\begin{array}{l}\text { Doxepin } \\
\text { hydrochloride } \\
\text { tablets }\end{array}$ & $58 / 58$ & $\begin{array}{l}59.45 \pm \\
9.03 / \\
58.16 \pm 8.14\end{array}$ & $\begin{array}{l}\text { HAM-D } \\
\text { scores } \geq 17\end{array}$ & $4 w$ & $\begin{array}{l}\text { HAM-D } \\
\text { scores }\end{array}$ & None & $\begin{array}{l}\text { Low } \\
\text { quality }\end{array}$ \\
\hline Li 2015 & {$[25]$} & $\begin{array}{l}\text { EA (LI4, LR3, } \\
\text { continuous }\end{array}$ & $\begin{array}{l}\text { Fluoxetine } \\
(20 \mathrm{mg} / \mathrm{d})\end{array}$ & $11 / 10$ & $\begin{array}{l}62.56 \pm 6.85 \\
66.42 \pm 6.25\end{array}$ & $\begin{array}{l}\operatorname{HAM}-D_{17} \geq \\
18\end{array}$ & $8 w$ & HAM-D 17 & NR & $\begin{array}{l}\text { Moderate } \\
\text { quality }\end{array}$ \\
\hline
\end{tabular}


Table 1 Characteristics of RCTs Included in the Review and Summary of Trial Quality Assessment (Continued)

\begin{tabular}{|c|c|c|c|c|c|c|c|c|c|c|}
\hline \multirow[t]{2}{*}{ Source } & \multirow{2}{*}{$\begin{array}{l}\text { Reference } \\
\text { No. }\end{array}$} & \multicolumn{2}{|l|}{ Intervention } & \multirow{2}{*}{$\begin{array}{l}\text { Sample } \\
\text { Size (T/ } \\
\text { C), } \\
\text { Dropout } \\
\text { (T/C) }\end{array}$} & \multirow[t]{2}{*}{ Age $(T / C)$} & \multirow{2}{*}{$\begin{array}{l}\text { Depression } \\
\text { Definition }\end{array}$} & \multirow[t]{2}{*}{ Duration } & \multirow{2}{*}{$\begin{array}{l}\text { Outcome } \\
\text { (s) }\end{array}$} & \multirow[t]{2}{*}{ Adverse events } & \multirow{2}{*}{$\begin{array}{l}\text { Overall } \\
\text { quality } \\
\text { for each } \\
\text { trial }\end{array}$} \\
\hline & & Treatment & Control & & & & & & & \\
\hline & & $\begin{array}{l}\text { waves } 2 \mathrm{~Hz} \text {, } \\
20 \mathrm{~min} / \mathrm{d} \text { ) }\end{array}$ & & & & & & & & \\
\hline $\begin{array}{l}\text { Chu } \\
2007\end{array}$ & [31] & $\begin{array}{l}\text { EA (GV20, } \\
\text { DU26, GV29, } \\
\text { PC6, sparse- } \\
\text { dense waves } \\
6-8 \mathrm{~Hz}, 30 \\
\mathrm{~min} / \mathrm{d})\end{array}$ & $\begin{array}{l}\text { Fluoxetine } \\
(20 \mathrm{mg} / \mathrm{d})\end{array}$ & $36 / 36$ & $\begin{array}{l}54-78 / 58- \\
72\end{array}$ & $\begin{array}{l}\text { HAM-D } \\
\text { scores } \geq 8\end{array}$ & $8 w$ & $\begin{array}{l}\text { HAM-D } \\
\text { scores }\end{array}$ & $\begin{array}{l}\text { C: } 3 \text { cases of dry } \\
\text { mouth, } 3 \text { cases } \\
\text { of dizziness, } 2 \\
\text { cases of } \\
\text { drowsiness. }\end{array}$ & $\begin{array}{l}\text { Moderate } \\
\text { quality }\end{array}$ \\
\hline $\begin{array}{l}\text { Sun } Y \\
b_{2} 2015\end{array}$ & [37] & $\begin{array}{l}\text { EA (the } \\
\text { midnight- } \\
\text { noon ebb- } \\
\text { flow theory, } \\
\text { continuous } \\
\text { wave, } 2 \mathrm{~Hz} \text {, } \\
30 \mathrm{~min} / \mathrm{d} \text { ) }\end{array}$ & $\begin{array}{l}\text { Fluoxetine } \\
\text { (20 mg/d) }\end{array}$ & $31 / 31$ & $\begin{array}{l}67 \pm 4 / 69 \pm \\
5\end{array}$ & $\begin{array}{l}\text { HAM-D }{ }_{17} \geq \\
17\end{array}$ & $6 m$ & HAM-D 17 & $\begin{array}{l}\text { C: } 2 \text { cases of } \\
\text { intermittent } \\
\text { headache, } 2 \\
\text { cases of loss of } \\
\text { appetite, } 2 \text { cases } \\
\text { of nausea. }\end{array}$ & $\begin{array}{l}\text { High } \\
\text { quality }\end{array}$ \\
\hline
\end{tabular}

$T$ treatment group, $C$ control group, EA electroacupuncture, HAM-D Hamilton Rating Scale for Depression, HAM-D 17 HAM-D scale-17 items, HAM-D 24 HAM-D scale24 items, NR Not reporting

${ }^{\mathrm{a} C}$ Conventional treatment includes psychological counselling, physical rehabilitation, antidepressants and basic treatment; ${ }^{\mathrm{b}}$ This trial contained 3 groups: acupuncture, acupuncture combined with antidepressants and antidepressants

methods. However, two studies [24, 36] did not provide details regarding random sequence generation and allocation concealment, so they were both considered high risk. Three trials [30, 33, 37] that described adequate methods used for allocation concealment were ranked as low risk. Twelve studies $[16,17,23,25-29,31,32,34,35]$ had unclear risks in terms of allocation concealment. One study [30] reported a double-blinded trial design, and two additional studies $[26,33]$ reported that they blinded participants and outcome assessment reviewers; accordingly, they were ranked as low risk. All studies had dropout rates of less than $20 \%$, so they were all rated as low risk in this aspect. Seven studies [24-28, 32, 35] failed to report adverse events; accordingly, they ranked as high risk in terms of reporting bias. Overall, three studies [30,33, 37] were rated as high quality, two [24, 36] were rated as low quality, and the others were rated as moderate quality. Figure 2 shows the assessment of the risk of bias, and Table 1 shows the quality of the included trials.

\section{Outcomes}

\section{Ham- $D_{17}$}

A meta-analysis of 7 studies [25, 26, 28, 30, 33, 34, 37] included 509 participants who used the HAM-D 17 to examine depression using a random-effects model. As shown in Fig. 3 studies [26, 28, 37] compared the combination of acupuncture/electroacupuncture and conventional treatment with conventional treatment and found that participants who received acupuncture exhibited significantly lower HAM- $D_{17}$ scores than those in the control group (MD, -5.08 [95\% CI, -6.48 to -3.67$]$, $\left.I^{2}=0 \%\right)$. One trial [37] recruited participants with mild depression, and a sensitivity analysis in which this trial was excluded showed that the results did not change (Supplemental Table 5). Five studies [25, 30, 33, 34, 37] compared acupuncture/electroacupuncture (+placebo) with antidepressants (+sham acupuncture), and the subgroup results demonstrated that there was no significant difference (MD, -0.43 [95\% CI, -1.61 to 0.75$], I^{2}=$ 51\%). Because Qian 2015 [33] and Li 2011 [30] combined treatment with placebo, we excluded them in the sensitivity analysis, and the result was overturned (Supplemental Table 5).

\section{Ham- $D_{24}$}

We identified 4 studies $[16,24,27,35]$ that made use of HAM- $\mathrm{D}_{24}$ tool to measure depression (Fig. 4). Two studies $[16,35]$ compared the combination of acupuncture/electroacupuncture and conventional treatment with conventional treatment, and a pooled analysis demonstrated that acupuncture led to significant reductions in HAM- $\mathrm{D}_{24}$ scores relative to sham acupuncture (MD, $-9.72[95 \% \mathrm{CI},-14.54$ to -4.91$\left.], I^{2}=65 \%\right)$. Another two studies compared acupuncture/electroacupuncture with antidepressants, and there was no significant difference in the risk of depression between these treatments (MD, -3.09 [95\% CI, -10.81 to 4.63$], I^{2}=90 \%$ ).

\section{Ham-d}

Six studies [17, 23, 29, 31, 32, 36] did not report which version of the HAM-D they used (Fig. 5). Pooled results from 2 studies [17, 23] showed that the reduction in HAM-D score was significantly associated with a combination of acupuncture and conventional treatment 


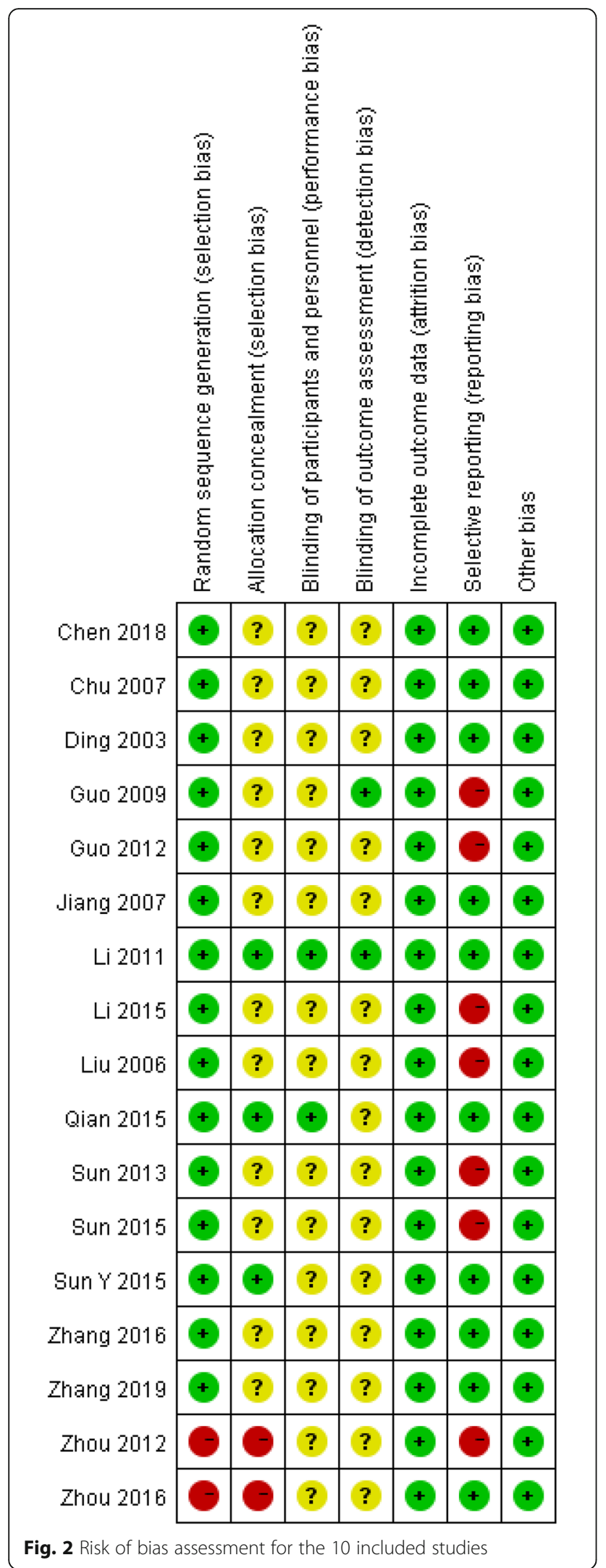

when compared with conventional treatment (MD, 2.72 [ $95 \% \mathrm{CI},-3.61$ to -1.82 ], I $2=0 \%$ ). Four studies $[29,31,32,36]$ showed a greater association between acupuncture and HAM-D than between antidepressants and HAM-D, though the studies had substantial heterogeneity (MD, -1.55 [95\% CI, -4.36 to 1.26$], I^{2}=95 \%$ ). In the sensitivity analyses, excluding the low-quality study [36] and combining studies by degree of depression did not change the results (Supplemental Table 5).

\section{Adverse events}

Ten studies [16, 17, 23, 29-31, 33, 34, 36, 37] reported adverse events during the study period (Fig. 6). Data from 4 RCTs [29-31, 34] were abstract to show that the occurrence of adverse events was significantly associated with acupuncture when compared with antidepressants (RR, 0.16 [95\% CI, 0.07 to 0.39 ] $I^{2}=35 \%$ ). When $\mathrm{Li}$ 2011 was excluded [30], sensitivity analyses showed that the results were not altered (Supplemental Table 5). There was no significant difference between acupuncture combined with conventional treatment and conventional treatment (RR, 0.63 [95\% CI, 0.21 to 1.83 ], $I^{2}=38 \%$ ). In addition, the adverse events reported were mild and disappeared without medical or specific intervention.

\section{Quality of evidence}

According to the GRADE system, the 8 outcomes were ranked as having low- or very low-quality evidence. The factors driving this quality assessment might be inappropriate randomization methods, lack of allocation concealment, and small sample size. The details of the evidence quality assessment are shown in Table 2.

\section{Discussion}

\section{Main findings}

This MA of 17 RCTs involving 1402 patients with PSD illustrated that acupuncture combined with conventional treatment could significantly reduce the PSD degree, regardless of which versions of the HAM-D score is used, compared with conventional treatment, although the studies have a low to very low level of evidence. However, of the comparison of acupuncture and antidepressants was inconclusive, and the quality of evidence was low to very low. We refined the different groups to determine the incidence of adverse events. Acupuncture was safer than antidepressants, and the combination of acupuncture and conventional treatment had no significant difference in adverse events compared with conventional treatment.

\section{Comparison to previous studies}

This nonblinded review of trials highlights that the combination of acupuncture and conventional treatment had a significant effect on HAM-D scores compared with 


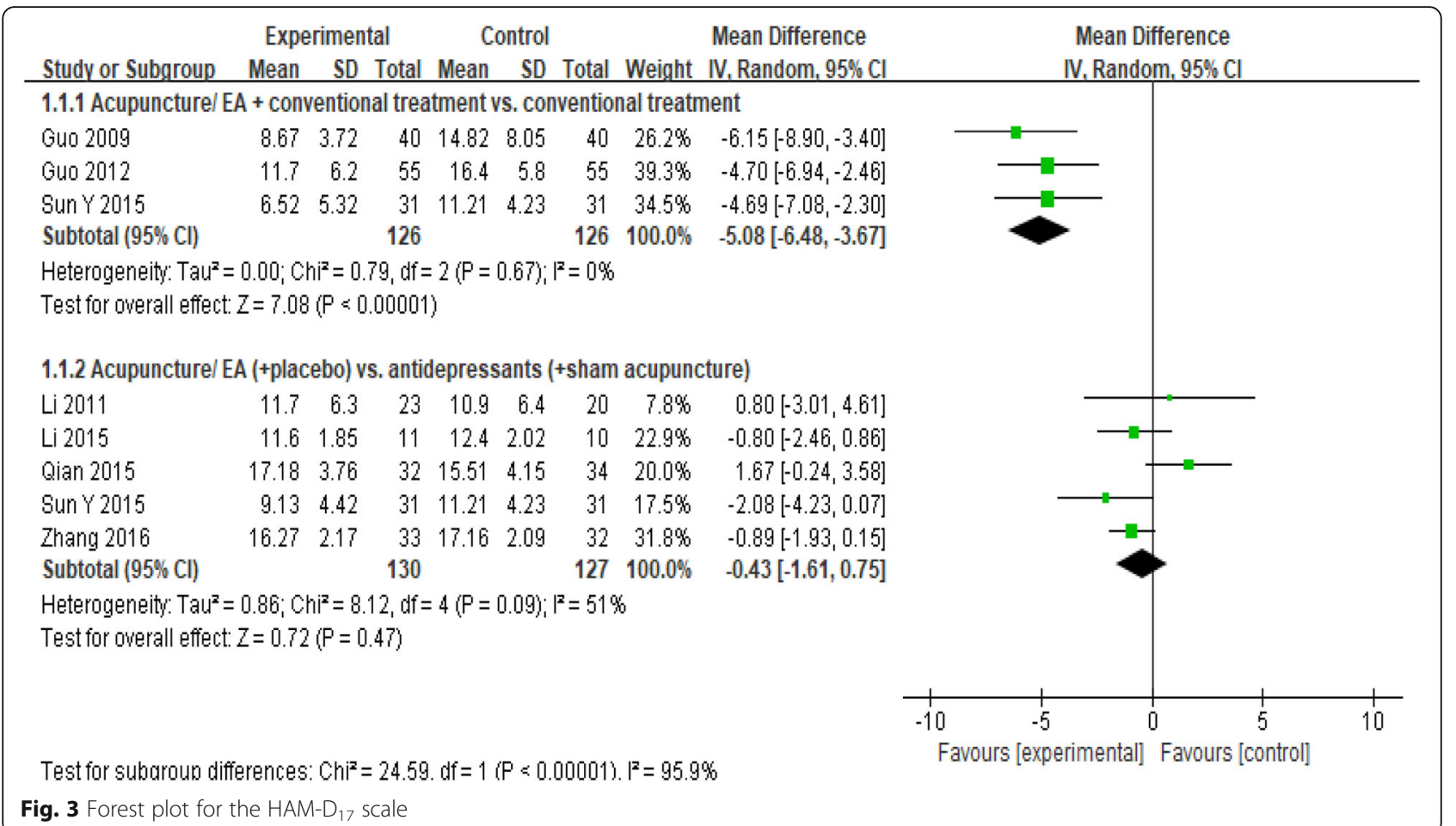

conventional treatment, and the result was consistent regardless of the degree of depression, which is consistent with findings in previous studies and reviews [39, 40] and helps to increase confidence in our findings. Although evidence from open-label studies illustrates the increased risk of bias from nonblinding, the use of nonblinded pragmatic trial procedures to maximize the applicability of treatment effectiveness in real-world situations has increased in recent years [41, 42]. Apart from this, we found that acupuncture has no effect on reducing the degree of PSD compared with antidepressants; these results are contrary to those reported in a previous

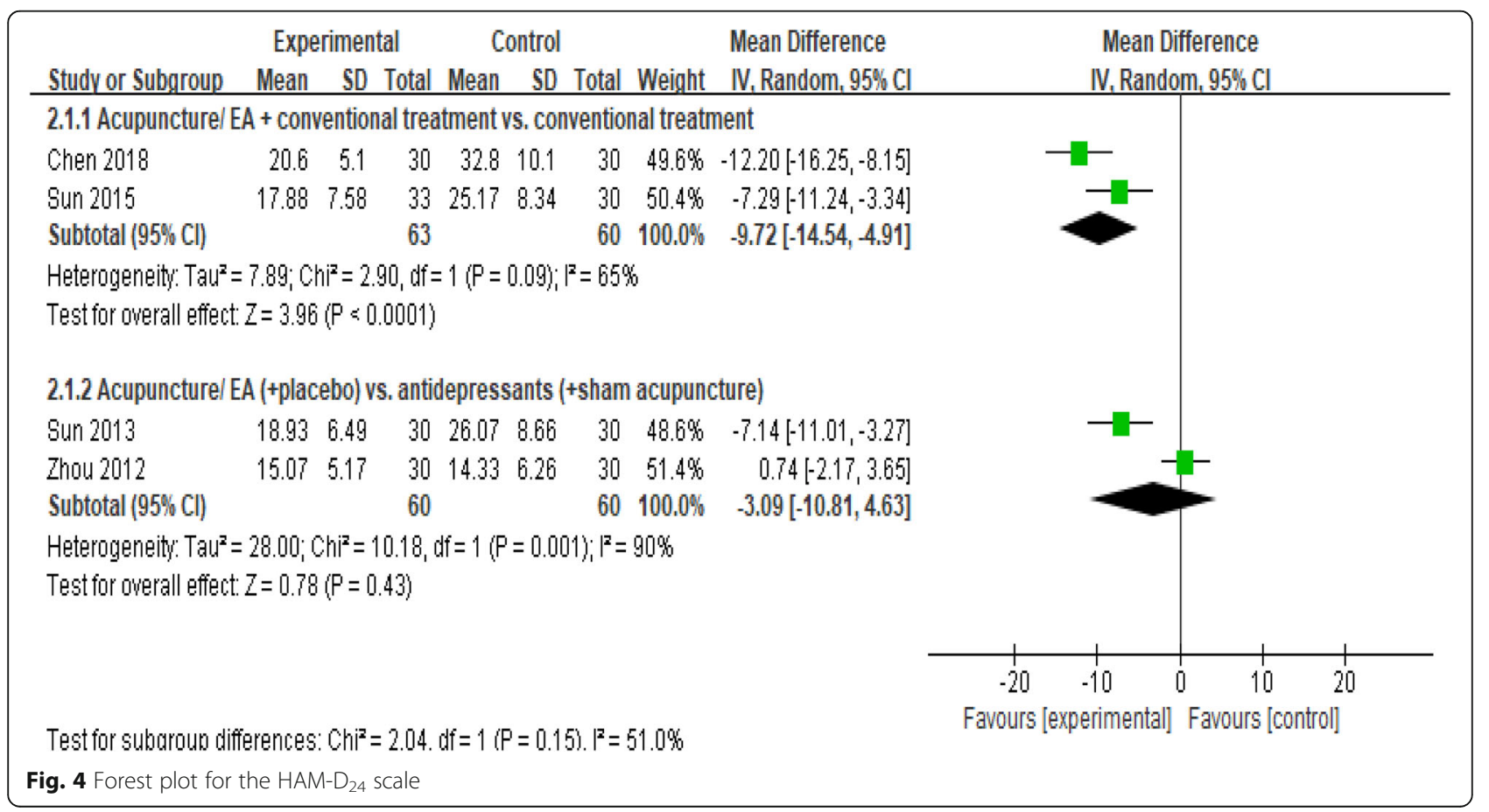




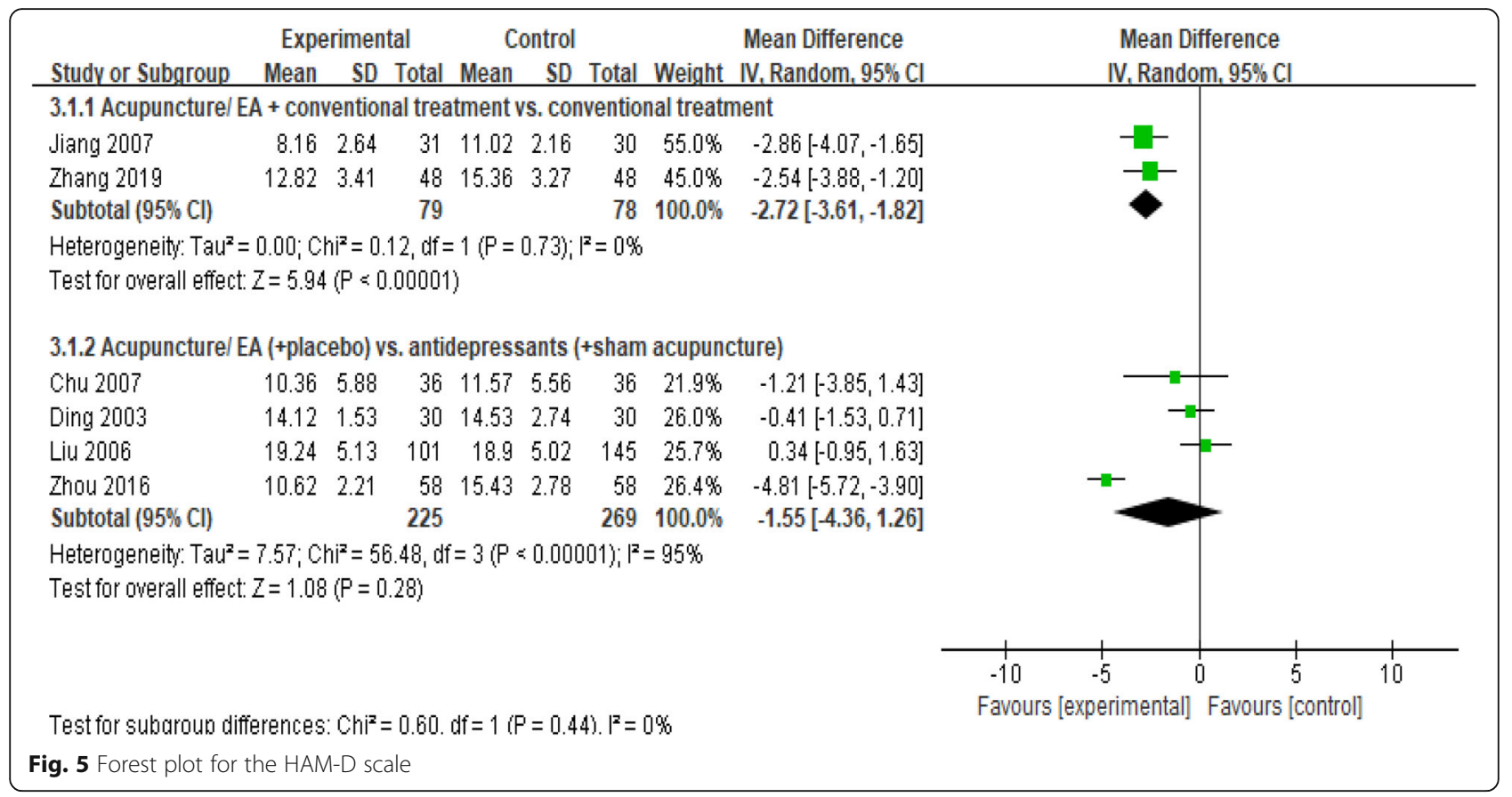

study [43]. When exploring the reasons for this discrepancy, we found that the placebo effect [44] is an important factor that cannot be ignored. Placebo interventions aim to intentionally utilize the placebo effect by increasing patients' expectations [45]. A meta-analysis demonstrated that sham acupuncture (SA) had a larger effect than other placebos [46]. Thus, it was very difficult to evaluate the effect of SA compared to that of real acupuncture [47]. Therefore, when we removed the placebo-controlled study, we found that the efficacy of acupuncture for PSD might be better than that of antidepressants.

In this review, we also focused on the differences in the estimated efficacy of acupuncture on PSD using different versions of the HAM-D scale. Our review included the HAM-D $17, H A M-D_{24}$ and other versions,

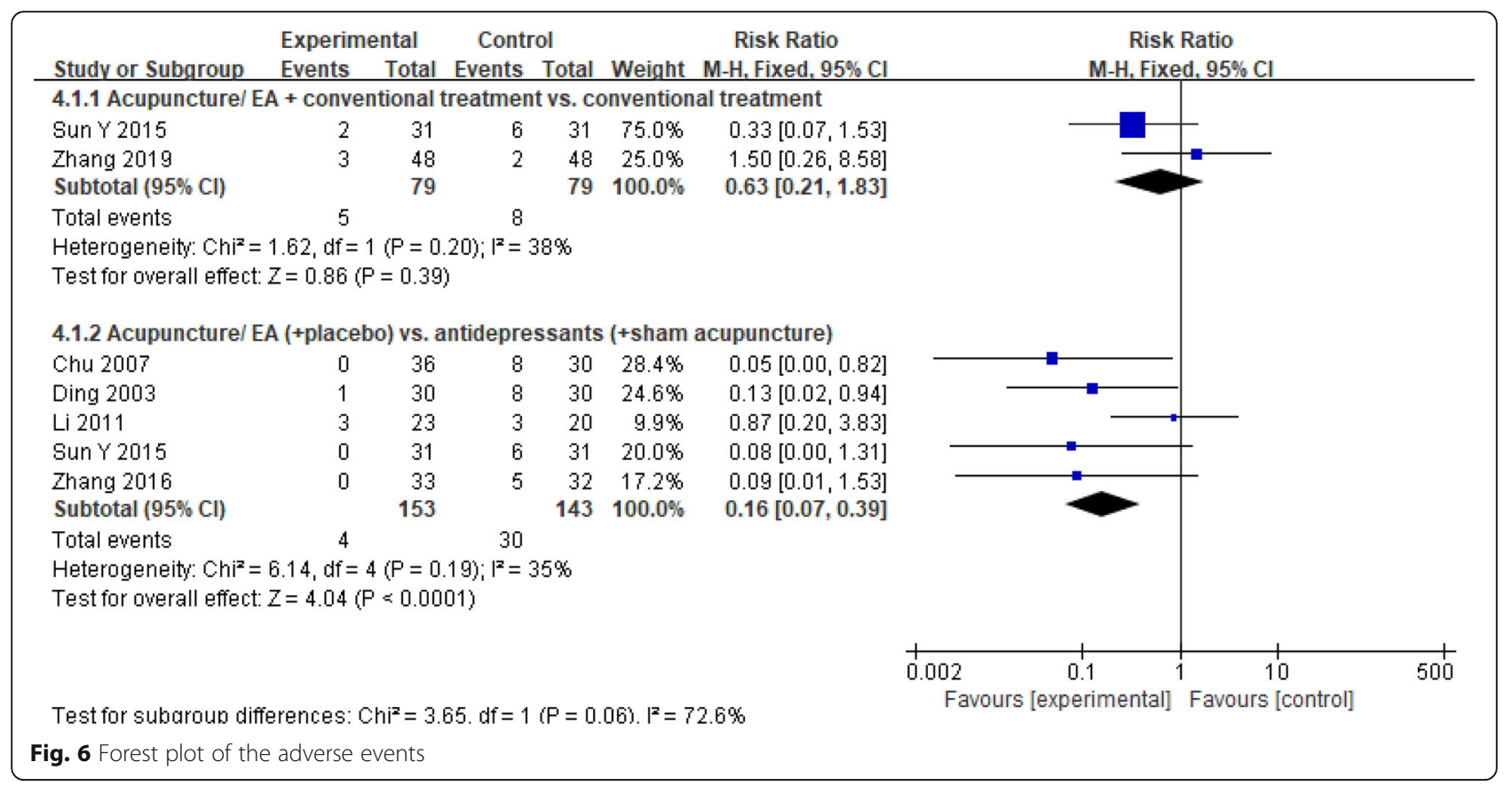


Table 2 Quality of evidence of included reviews according to GRADE

\begin{tabular}{|c|c|c|c|c|c|c|c|c|c|c|}
\hline \multicolumn{7}{|c|}{ Certainty Assessment } & \multicolumn{2}{|c|}{ No. of Patients } & \multirow[b]{2}{*}{$\begin{array}{l}\text { Effect }(95 \% \\
\text { CL) }\end{array}$} & \multirow[b]{2}{*}{ Certainty } \\
\hline $\begin{array}{l}\text { No. of } \\
\text { Studies }\end{array}$ & $\begin{array}{l}\text { Reference } \\
\text { No. }\end{array}$ & $\begin{array}{l}\text { Risk of } \\
\text { Bias }\end{array}$ & Inconsistency & Indirectness & Imprecision & $\begin{array}{l}\text { Publication } \\
\text { Bias }\end{array}$ & $\begin{array}{l}\text { Treatment } \\
\text { Group }\end{array}$ & $\begin{array}{l}\text { Control } \\
\text { Group }\end{array}$ & & \\
\hline \multicolumn{11}{|c|}{ Acupuncture/EA (+placebo) compared to antidepressants (+sham acupuncture) } \\
\hline \multicolumn{11}{|c|}{ HAM-D 17} \\
\hline \multicolumn{2}{|c|}{$\begin{array}{c}{[25,30,33,34} \\
37]\end{array}$} & $\underset{\mathrm{a}}{\text { serious }}$ & serious ${ }^{b}$ & not serious & serious $^{c}$ & none & 130 & 127 & $\begin{array}{l}\text { MD }-0.43 \\
(-1.61,0.75)\end{array}$ & $\begin{array}{l}\text { ๑OOOVERY } \\
\text { LOW }\end{array}$ \\
\hline \multicolumn{11}{|c|}{ HAM-D 24} \\
\hline \multicolumn{2}{|c|}{$[24,27]$} & $\begin{array}{l}\text { serious } \\
\text { a }\end{array}$ & serious $^{d}$ & not serious & $\underset{c}{\text { very serious }}$ & none & 60 & 60 & $\begin{array}{l}M D-3.09(- \\
10.81,4.63)\end{array}$ & $\begin{array}{l}\text { ФOOOVERY } \\
\text { LOW }\end{array}$ \\
\hline \multicolumn{11}{|c|}{ HAM-D } \\
\hline \multicolumn{2}{|c|}{$[29,31,32,36]$} & $\begin{array}{l}\text { serious } \\
\text { a }\end{array}$ & serious ${ }^{\mathrm{e}}$ & not serious & not serious & none & 225 & 269 & $\begin{array}{l}\mathrm{MD}-1.55 \\
(-4.36,1.26)\end{array}$ & $\oplus \oplus \circ O L O W$ \\
\hline \multicolumn{11}{|c|}{ Adverse events } \\
\hline \multicolumn{2}{|c|}{$[29-31,34,37]$} & $\underset{a}{\text { serious }}$ & not serious & serious $^{f}$ & serious $^{c}$ & none & $\begin{array}{l}4 / 153 \\
(2.6 \%)\end{array}$ & $\begin{array}{l}30 / 143 \\
(21 \%)\end{array}$ & $\begin{array}{l}\text { RR } 0.16(0.07 \\
0.39)\end{array}$ & $\begin{array}{l}\text { ФOOOVERY } \\
\text { LOW }\end{array}$ \\
\hline \multicolumn{11}{|c|}{ Acupuncture/EA + conventional treatment compared to conventional treatment } \\
\hline \multicolumn{11}{|c|}{$\mathrm{HAM} \mathrm{D}_{17}$} \\
\hline \multicolumn{2}{|c|}{$[26,28,37]$} & $\underset{a}{\text { serious }}$ & not serious & not serious & serious $^{c}$ & none & 126 & 126 & $\begin{array}{l}M D-5.08(-6.4 \\
-3.67)\end{array}$ & $\oplus \oplus \circ O L O W$ \\
\hline \multicolumn{11}{|c|}{$\mathrm{HAM}-\mathrm{D}_{24}$} \\
\hline \multicolumn{2}{|c|}{$[16,35]$} & $\begin{array}{l}\text { serious } \\
\text { a }\end{array}$ & serious $^{\mathrm{g}}$ & not serious & $\begin{array}{l}\text { very serious } \\
\text { c }\end{array}$ & none & 63 & 60 & $\begin{array}{l}M D-9.72 \\
(-14.54,-4.91)\end{array}$ & $\begin{array}{l}\text { ФOOOVERY } \\
\text { LOW }\end{array}$ \\
\hline \multicolumn{11}{|c|}{ HAM-D } \\
\hline \multicolumn{2}{|c|}{$[17,24]$} & $\underset{a}{\text { serious }}$ & not serious & not serious & $\begin{array}{l}\text { very serious } \\
\text { c }\end{array}$ & none & 79 & 78 & $\begin{array}{l}M D-\mathbf{2 . 7 2} \\
(-3.61,-1.82)\end{array}$ & $\begin{array}{l}\text { ФOOOVERY } \\
\text { LOW }\end{array}$ \\
\hline \multicolumn{11}{|c|}{ Adverse events } \\
\hline 2 & {$[17,37]$} & $\begin{array}{l}\text { serious } \\
\text { a }\end{array}$ & not serious & serious ${ }^{f}$ & $\begin{array}{l}\text { very serious } \\
c\end{array}$ & none & $5 / 79(6.3 \%)$ & $\begin{array}{l}8 / 79 \\
(10.1 \%)\end{array}$ & $\begin{array}{l}\text { RR } 0.66(0.15 \\
2.89)\end{array}$ & $\begin{array}{l}\text { ФOOOVERY } \\
\text { LOW }\end{array}$ \\
\hline
\end{tabular}

$C l$ confidence interval, MD mean difference, $R R$ relative risk, HAM-D Hamilton Depression Scale, HAM- $D_{17}$ HAM-D scale-17 items, HAM- $D_{24}$ HAM-D scale-24 items ${ }^{\mathrm{a}}$ This trial had a large bias in randomization, allocation concealment, blinding, or selective reporting; $\left.{ }^{\mathrm{b}}\right|^{2}=51 \%$; ${ }^{\mathrm{C}}$ Small sample size; ${ }^{\mathrm{d}} \mathrm{I}^{2}=90 \%$; ${ }^{\mathrm{e}}{ }^{2}=95 \%$; ${ }^{\mathrm{f}}$ This is a secondary outcome; $\left.{ }^{9}\right|^{2}=65 \%$

which are all commonly used clinically [12]. The HAM$\mathrm{D}_{17}$ is sensitive enough to discriminate different levels of depression severity and a cut-off score of $<8$ defines no depression $[48,49]$. For the HAM- ${ }_{24}$, a cut-off score of less than 10 is used; this version has expanded items assessing worthlessness, symptoms of helplessness, hopelessness and cognitive impairment [48], though it was less sensitive with regard to discriminating antidepressants and placebo than the HAM- $\mathrm{D}_{17}$ [50]. A study [51] has indicated that when compared with antidepressants, acupuncture had lower scores on the HAM- $\mathrm{D}_{17}$ than on the HAM- $\mathrm{D}_{24}$. In our review, the comparison of acupuncture and antidepressants showed no difference in efficacy when different versions of the HAM-D were used, so we could not make a more appropriate version to evaluate the efficacy of acupuncture on PSD. In our study, acupuncture combined with conventional treatments compared with conventional treatments for PSD was the most effective when depression was measured using the HAM- $\mathrm{D}_{24}$ scale, followed by the HAM- $\mathrm{D}_{17}$ scale, finally, the HAM-D scale. There exists a weak relationship between the HAM-D scale version used to evaluate the effect of acupuncture on depression and the strength of the association, yet this relationship depended on the intervention used. In addition, some researchers have proposed that the Post-Stroke Depression Scale (PSDS) is a valid, reliable and specific tool to evaluate PSD [52], and some hold that the HAM- $\mathrm{D}_{6}$ could be a new indicator to evaluate the effect of acupuncture on PSD because it aims to investigate the discrimination between active treatment and placebo [50, 53]. Therefore, deciding which version of the HAM-D scale to use remains a challenge, but in terms of redundancy, the HAM- $\mathrm{D}_{24}$ clearly carries more load than the HAM- $D_{17}$ [50], and in terms of usage, the HAM- $\mathrm{D}_{17}$ $(41 \%)$ was more common in our review than HAM- $\mathrm{D}_{24}$ (23\%).

A recent series of studies of antidepressants have claimed that antidepressant effectiveness is beyond dispute, but its side effects should not be ignored [54-56]. 
In our review, we prove that the combination of acupuncture and antidepressants has no significant effect on adverse events compared with antidepressants alone. However, the incidence of adverse events associated with acupuncture was apparently lower than that associated with antidepressants, and the results of sensitivity analyses were not altered when studies with placebo were excluded. This suggests that acupuncture may be a safer treatment for PSD. This finding is similar to that of a previous review [11].

One common issue in acupuncture programs is the lack of standardization of point selection, duration, frequency, number of sessions and use of electroacupuncture [57]. The 17 RCTs evaluated here revealed that the most commonly selected points were GV20 (65\%), PC6 (53\%), and LR3 (47\%), which is different from the most recent study [11]. Additionally, our review varied greatly in terms of acupuncture duration and the number of sessions, ranging from $15 \mathrm{~min}$ to $30 \mathrm{~min}$ of duration and from 1 month to 6 months in terms of number of sessions. There is no clear concept of an appropriate acupuncture dose and how much treatment is needed for a given situation. The lack of agreement on the optimal acupuncture treatment for any particular condition may partly impact the acupuncture effect [58].

\section{Strengths and limitations}

There are some advantages of this review. To ensure the quality of the included studies, we used more rigorous inclusion criteria; for example, all Chinese RCTs included in this review were published in the PKU journal. In addition, considerable effort was made to carry out an extensive literature search, including relevant SRs and MAs, as well as a supplemental search of recent RCTs. For intervention methods, we made efforts to analyse the combination of acupuncture and conventional treatment compared with conventional treatment or acupuncture compared with antidepressants. In addition, we investigated the effects of acupuncture for PSD using different HAM-D versions, which could provide a clinical reference for further study.

This review has several limitations. First, due to the quantities of included trials, we could not explore whether different acupuncture durations could impact the results. Second, this review indicated that acupuncture for PSD can largely reduce the degree of depression, but we did not find a suitable clinically important difference (MCID) for the HAM-D, which limits our ability to determine the clinical effectiveness. Third, some of the studies included in this review may reduce the reliability of the conclusions due to factors such as a lack of allocation concealment, small sample size, or unclear study blinding.

\section{Conclusions}

This review strengthens the evidence that acupuncture could reduce the degree of PSD. However, there is still a lack of moderate and high quality evidence to support this conclusion. Further methodologically rigorous and adequately powered primary studies are necessary to assess the effect of acupuncture on PSD.

\section{Abbreviations}

PSD: Post-stroke depression; RCTs: Randomized clinical trials; SRs: Systematic reviews; MAs: Meta-analyses; GRADE: Grades of Recommendation,

Assessment, Development and Evaluation; 5-HT: 5-hydroxytryptamine; HAMD: Hamilton Rating Scale for Depression; CNKI: China National Knowledge Infrastructure; CBM: Chinese Biomedical Medicine disc; CQVIP: Chongqing VIP; Cl: Confidence interval; MD: Mean difference; RR: Relative risk; PSDS: PostStroke Depression Scale; T: Treatment group; C: Control group;

EA: Electroacupuncture

\section{Supplementary Information}

The online version contains supplementary material available at https://doi. org/10.1186/s12906-021-03277-3.

Additional file 1: Table S1. Search strategy to identify RCTs in PubMed. Table S2. Search strategy to identify meta-analysis in PubMed. Table S3. Randomized trials included in systematic reviews or metaanalyses evaluating acupuncture and PSD. Table S4. Excluded trials and reasons for exclusion. Table S5. Results of sensitivity analyses excluding the listed trials. Table S6. The acupoints and their frequency of use in the included studies. Table S7. The treatment time and frequency in the included studies. Table S8. The treatment time and frequency in the included studies.

Acknowledgements

Not to be declared.

\section{Authors' contributions}

WDS, WM and RL provided the concept and design. WM and RL searched the literature databases. KZ, GWC and QYT selected eligible studies and extracted the data. $\mathrm{RL}$ and $\mathrm{KZ}$ evaluated risk of bias. $\mathrm{RL}$ and QYT conducted the GRADE evaluation. WM and RL drafted the first version of the manuscript. WDS and WM supervised the project as supervisors. All authors approved the final submission.

\section{Funding}

The study was supported by grants from the Science and Technology Commission of Shanghai Municipality, China (18401970601). The Funding recipient WDS provided the concept and design of the manuscript, and made suggestions for modification.

\section{Availability of data and materials}

The datasets generated and analysed during the current study are available in the 7 databases (details in the study selection section).

\section{Declarations}

Ethics approval and consent to participate

Not applicable.

Consent for publication

Not applicable.

\section{Competing interests}

The authors declare that they have no competing interests.

\section{Author details}

${ }^{1}$ Department of Acupuncture, Shuguang Hospital, Shanghai University of Traditional Chinese Medicine, Shanghai 201203, China. ${ }^{2}$ Institute of 
Acupuncture and Anesthesia, Shuguang Hospital, Shanghai University of Traditional Chinese Medicine, Shanghai 201203, China.

\section{Received: 24 November 2020 Accepted: 17 March 2021 Published online: 01 April 2021}

\section{References}

1. Johnson CO, Nguyen M, Roth GA, Nichols E, Alam T, Abate D, et al. Global, regional, and national burden of stroke, 1990-2016: a systematic analysis for the global burden of disease study 2016. Lancet Neurol. 2019;18(5):439-58. https://doi.org/10.1016/S1474-4422(19)30034-1.

2. Das J, Rajanikant GK. Post stroke depression: the sequelae of cerebral stroke. Neurosci Biobehav Rev. 2018;90:104-14. https://doi.org/10.1016/j.neubiorev.2 018.04.005.

3. Schöttke $\mathrm{H}$, Giabbiconi CM. Post-stroke depression and post-stroke anxiety: prevalence and predictors. Int Psychogeriatr. 2015;27(11):1805-12. https:// doi.org/10.1017/S1041610215000988.

4. Žikić TR, Divjak I, Jovićević M, Semnic M, Slankamenac P, Žarkov M, et al. The effect of post stroke depression on functional outcome and quality of life. Acta Clin Croatica. 2014;53(3):294-301.

5. Shi YZ, Xiang YT, Yang Y, Zhang N, Wang S, Ungvari GS, et al. Depression after minor stroke: the association with disability and quality of life--a 1-year follow-up study. Int J Geriatr Psychiatry. 2016;31(4):421-7. https://doi.org/1 0.1002 /gps.4353.

6. Blöchl M, Meissner S, Nestler S. Does depression after stroke negatively influence physical disability? A systematic review and meta-analysis of longitudinal studies. J Affect Disord. 2019;247:45-56. https://doi.org/10.1016/ j.jad.2018.12.082

7. Cai W, Mueller C, Li YJ, Shen WD, Stewart R. Post stroke depression and risk of stroke recurrence and mortality: a systematic review and meta-analysis. Ageing Res Rev. 2019;50:102-9. https://doi.org/10.1016/j.arr.2019.01.013.

8. Hoirisch-Clapauch S, Nardi AE. Antidepressants: bleeding or thrombosis? Thromb Res. 2019;181:S23-S8. https://doi.org/10.1016/S0049-3848(19)303627

9. Trajkova S, d'Errico A, Soffietti R, Sacerdote C, Ricceri F. Use of antidepressants and risk of incident stroke: a systematic review and Metaanalysis. Neuroepidemiology. 2019;53(3-4):142-51. https://doi.org/10.1159/ 000500686.

10. Ladwig S, Zhou Z, Xu Y, Wang X, Chow CK, Werheid K, et al, Comparison of treatment rates of depression after stroke versus myocardial infarction: a systematic review and Meta-analysis of observational data. Psychosom Med. 2018;80(8):754-63. https://doi.org/1 0.1097/PSY.0000000000000632.

11. Li XB, Wang J, Xu AD, Huang JM, Meng LQ, Huang RY, et al. Clinical effects and safety of electroacupuncture for the treatment of post-stroke depression: a systematic review and meta-analysis of randomised controlled trials. Acupunct Med. 2018;36(5):284-93. https://doi.org/10.1136/acupmed-2 016-011300.

12. Zhang J, Chen J, Chen J, Li X, Lai X, Zhang S, et al. Early filiform needle acupuncture for poststroke depression: a meta-analysis of 17 randomized controlled clinical trials. Neural Regen Res. 2014;9(7):773-84. https://doi. org/10.4103/1673-5374.131590

13. Zhang GC, Fu WB, Xu NG, Liu JH, Zhu XP, Liang ZH, et al. Meta analysis of the curative effect of acupuncture on post-stroke depression. J Tradit Chin Med. 2012;32(1):6-11. https://doi.org/10.1016/S0254-6272(12)60024-7.

14. Zhang ZJ, Chen HY, Yip KC, Ng R, Wong VT. The effectiveness and safety of acupuncture therapy in depressive disorders: systematic review and metaanalysis. J Affect Disord. 2010;124(1-2):9-21. https://doi.org/10.1016/j.jad.2 009.07.005.

15. Zhang XY, Li YX, Liu DL, Zhang BY, Chen DM. The effectiveness of acupuncture therapy in patients with post-stroke depression: an updated meta-analysis of randomized controlled trials. Medicine (Baltimore). 2019; 98(22):e15894. https://doi.org/10.1097/MD.0000000000015894.

16. Chen A, Gao Y, Wang G, Li J, Shen W. Effect of early acupuncture intervention on post-stroke depression: a randomized controlled trial. Zhongguo Zhen Jiu. 2018;38(11):1141-4. https://doi.org/10.13703/j.0255-293 0.2018 .11 .001 .

17. Zhang DR, Peng X, Zhi Y. Therapeutic effect observation on acupuncture plus medication for post-stroke depression. J Acupuncture Tuina Sci. 2019; 17(1):31-6. https://doi.org/10.1007/s11726-019-1085-z.
18. Higgins JP. In: The Cochrane Collaboration, editor. Cochrane Handbook for Systematic Reviews of Interventions version5.1.0 (updated March 2011); 2011. Available from: http://www.training.cochrane.org/handbook.

19. Moher D, Liberati A, Tetzlaff J, Altman DG, The PRISMA Group. Preferred reporting items for systematic reviews and meta-analyses: the PRISMA statement. PLoS Med. 2009;6(7):e1000097. https://doi.org/10.1371/journal. pmed.1000097.

20. Helmreich I, Wagner S, Mergl R, Allgaier AK, Hautzinger M, Henkel V, et al. Sensitivity to changes during antidepressant treatment: a comparison of unidimensional subscales of the inventory of depressive symptomatology (IDS-C) and the Hamilton depression rating scale (HAM-D) in patients with mild major, minor or subsyndromal depression. Eur Arch Psychiatry Clin Neurosci. 2012:262(4):291-304.

21. Zhao JG, Zeng XT, Wang J, Liu L. Association between calcium or vitamin D supplementation and fracture incidence in community-dwelling older adults: a systematic review and Meta-analysis. JAMA. 2017;318(24):2466-82. https://doi.org/10.1001/jama.2017.19344.

22. Guyatt GH, Oxman AD, Vist GE, Kunz R, Falck-Ytter Y, Alonso-Coello P, et al. GRADE: an emerging consensus on rating quality of evidence and strength of recommendations. BMJ. 2008;336(7650):924-6. https://doi.org/10.1136/ bmj.39489.470347.AD.

23. Jiang ZY, Li G, Du LC, Cun LJ. Clinical study on the treatment of post-stroke deepression with electroacupuncture of "NIWAN BAZHEN" points. World J Acupuncture Moxibustion. 2007;17(01):11-6.

24. Zhou CX, Cui $X$, Hu YS, Zeng HL, Ni HH, Huang CS, et al. Effect of combined acupuncture with Chinese medicine on overall function of patients with post-stroke depression. J Acupuncture Tuina Sci. 2012;10(2):99-103. https:// doi.org/10.1007/s11726-012-0581-1.

25. Li JB, Ye XM, Cheng RD, Zhu GY, Yang T. Effect of Electroacupuncture on regional cerebral blood flow in patients with post-stroke depression. Chin J Rehabil Theory Pract. 2015;21(02):192-5.

26. Guo RY, Su L, Liu LA, Wang CX. Effects of Linggui Bafa on the therapeutic effect and quality of life in patients of post-stroke depression. Zhongguo Zhen Jiu. 2009:29(10):785-90.

27. Sun PY, Chu HR, Li PF, Wang Y, Li F, Xia JG, et al. Post-stroke depression treated with acupuncture therapy of dredging governor vessel and regulating mentality: a randomized controlled trial. Chin Acupuncture Moxibustion. 2013;33(01):3-7.

28. Guo XD, Wang ML, Zhao XW, Hou DM, Qi YM. Effects of rehabilitation training combined with acupuncture intervention on post-stroke depression and daily life activities. Chin J Phys Med Rehabil. 2012;34(09):711-2.

29. Ding Z, Yu GX. Clinical study on treatment of post-stroke depression with acupuncture of Du Meridian as Main therapy. J Beijing Univ TCM. 2003; 10(03):31-3.

30. Li HJ, Zhong BL, Fan YP, Hu HT. Acupuncture for post-stroke depression:a randomized controlled trial. Chin Acupuncture Moxibustion. 2011;31(01):3-6.

31. Chu YJ, Wang CY, Zhang H. Clinical observation of 72 patients with post-stroke depression treated with acupuncture. Chin J Gerontol. 2007;27(17):1720-1.

32. Liu SK, Zhao XM, Xi ZM. Incidence rate and acupuncture-moxibustion treatment of post-stroke depression. Chin Acupuncture Moxibustion. 2006; 26(07):472-4.

33. Qian X, Zhou X, You Y, Shu S, Fang F, Huang S, et al. Traditional Chinese acupuncture for Poststroke depression: a single-blind double-simulated randomized controlled trial. J Altern Complement Med. 2015;21(12):748-53. https://doi.org/10.1089/acm.2015.0084.

34. Zhang LH, Zhang YC, Wang YJ, Han YX, Li PP. Efficacy observation on Governor Vessel-regulating and brain-unblocking acupuncture for poststroke depression. J Acupuncture Tuina Sci. 2016;14(3):175-80.

35. Sun $P, C h u H, L i P$, Wang $T, P u F, W u$ J, et al. The effect of the acupuncture intervention of dredging governor vessel and regulating mentality for the medication treatment of post-stroke depression. Zhongguo Zhen Jiu. 2015; 35(8):753-7.

36. Zhou MY, Wu LX. Clinical effectiveness of acupuncture in patients with depression and observation on safety evaluation after stroke. Chin Arch Tradit Chin Med. 2016;34(02):502-4

37. Sun YT, Bao YH, Wang SL, Chu JM, Li LP. Efficacy on post-stroke depression treated with acupuncture at the acupoints based on ziwuliuzhu and prozac. Chin Acupuncture Moxibustion. 2015:35(02):119-22.

38. Zimmerman M, Martinez JH, Young D, Chelminski I, Dalrymple K. Severity classification on the Hamilton depression rating scale. J Affect Disord. 2013; 150(2):384-8. https://doi.org/10.1016/j.jad.2013.04.028. 
39. Xu RX, Chen J. Meta-analysis and sequential analysis of depression of acupuncture treatment for post-stroke depression patients. Chin J Gerontol. 2019;39(23):5722-6.

40. Li XH, Chen JQ, Hu YN, Wang HT, Wang SX. Meta-analysis of the combination of acupuncture and western medicine and western medicine on the improvement of depression in patients with post-stroke depression. Shanxi Chin Med. 2012;33(09):1263-7.

41. He Y, Guo X, May BH, Zhang AL, Liu Y, Lu C, et al. Clinical evidence for Association of Acupuncture and Acupressure with Improved Cancer Pain: a systematic review and Meta-analysis. JAMA Oncol. 2019;6:271-8.

42. Sox HC, Lewis RJ. Pragmatic trials: practical answers to "real world" questions. JAMA. 2016;316(11):1205-6. https://doi.org/10.1001/jama.2016.114 09.

43. Zhang W, Sun JH, Gao Y, Pei LX, Wu XL, Chen L, et al. System review on treating post-stroke depression with acupuncture. World J Acupuncture Moxibustion. 2014;24(02):52-9. https://doi.org/10.1016/S1003-5257(14)60026$\mathrm{X}$.

44. Meissner K, Fassler M, Rucker G, Kleijnen J, Hrobjartsson A, Schneider A, et al. Differential effectiveness of placebo treatments: a systematic review of migraine prophylaxis. JAMA Intern Med. 2013;173(21):1941-51. https://doi. org/10.1001/jamainternmed.2013.10391.

45. Hamberger J, Meissner K, Hinterberger T, Loew T, Weimer K. Placebo economics: a systematic review about the economic potential of utilizing the placebo effect. Front Psychiatry. 2019;10:653. https://doi.org/10.3389/ fpsyt.2019.00653.

46. Linde K, Niemann K, Meissner K. Are sham acupuncture interventions more effective than (other) placebos? A re-analysis of data from the Cochrane review on placebo effects. Forsch Komplementmed. 2010;17(5):259-64. https://doi.org/10.1159/000320374.

47. Yuan QL, Wang P, Liu L, Sun F, Cai YS, Wu WT, et al. Acupuncture for musculoskeletal pain: a meta-analysis and meta-regression of shamcontrolled randomized clinical trials. Sci Rep. 2016:6(1):30675. https://doi. org/10.1038/srep30675.

48. Sharp R. The Hamilton rating scale for depression. Occup Med (Lond). 2015; 65(4):340. https://doi.org/10.1093/occmed/kqv043.

49. Carrozzino D, Patierno C, Fava GA, Guidi J. The Hamilton rating scales for depression: a critical review of Clinimetric properties of different versions. Psychother Psychosom. 2020;89(3):133-50. https://doi.org/10.1159/ 000506879.

50. Kyle PR, Lemming OM, Timmerby N, Sondergaard S, Andreasson K, Bech P. The validity of the different versions of the Hamilton depression scale in separating remission rates of placebo and antidepressants in clinical trials of major depression. J Clin Psychopharmacol. 2016;36(5):453-6. https://doi. org/10.1097/JCP.0000000000000557.

51. Zhang LL, Chu Q, Wang S, Lai H, Xie BB. Is sham acupuncture as effective as traditional Chinese acupuncture? It's too early to say. Chin J Integr Med. 2016;22(7):483-9. https://doi.org/10.1007/s11655-016-2458-5.

52. Yue Y, Liu R, Lu J, Wang X, Zhang S, Wu A, et al. Reliability and validity of a new post-stroke depression scale in Chinese population. J Affect Disord. 2015;174:317-23. https://doi.org/10.1016/j.jad.2014.11.031.

53. Ostergaard SD, Bech P, Miskowiak KW. Fewer study participants needed to demonstrate superior antidepressant efficacy when using the Hamilton melancholia subscale (HAM-D(6)) as outcome measure. J Affect Disord. 2016;190:842-5. https://doi.org/10.1016/j.jad.2014.10.047.

54. Gagne JJ, Patrick AR, Mogun H, Solomon DH. Antidepressants and fracture risk in older adults: a comparative safety analysis. Clin Pharmacol Ther. 2011; 89(6):880-7. https://doi.org/10.1038/clpt.2011.54.

55. Casilla-Lennon MM, Meltzer-Brody S, Steiner AZ. The effect of antidepressants on fertility. Am J Obstet Gynecol. 2016;215(3):314 e1-5.

56. Cipriani A, La Ferla T, Furukawa TA, Signoretti A, Nakagawa A, Churchill R, et al. Sertraline versus other antidepressive agents for depression. Cochrane Database Syst Rev. 2010;4:CD006117.

57. Dimitrova A, Murchison C, Oken B. Acupuncture for the treatment of peripheral neuropathy: a systematic review and Meta-analysis. J Altern Complement Med. 2017;23(3):164-79. https://doi.org/10.1089/acm.2016.0155.

58. White A, Cummings M, Barlas P, Cardini F, Filshie J, Foster NE, et al. Defining an adequate dose of acupuncture using a neurophysiological approach--a narrative review of the literature. Acupunct Med. 2008;26(2):111-20. https:// doi.org/10.1136/aim.26.2.111.

\section{Publisher's Note}

Springer Nature remains neutral with regard to jurisdictional claims in published maps and institutional affiliations.
Ready to submit your research? Choose BMC and benefit from:

- fast, convenient online submission

- thorough peer review by experienced researchers in your field

- rapid publication on acceptance

- support for research data, including large and complex data types

- gold Open Access which fosters wider collaboration and increased citations

- maximum visibility for your research: over $100 \mathrm{M}$ website views per year

At $\mathrm{BMC}$, research is always in progress.

Learn more biomedcentral.com/submissions 